\title{
Material science aspects of phase change optical recording
}

\author{
L K MALHOTRA, Y SRIPATHI and G B REDDY \\ Department of Physics, Indian Institute of Technology, New Delhi 110016, India
}

\begin{abstract}
Chalcogenide thin films are used as the recording medium for phase change-type optical memory discs. The films are switched between amorphous and crystalline states using the heat of a focussed laser beam. Large reflectivity differences between amorphous and crystalline states are then used to store and retrieve the information. An active chalcogenide layer for this purpose should have a high optical absorption coefficient ( $\alpha$ ), and good structural and thermal stability. It should be possibie to switch the chalcogenide layer between amorphous and crystalline states repeatedly within a short duration, the optical contrast should be high, and the material must have large cycling capability. Keeping the above requirements in mind, we have carried out systematic investigation of structural, optical and crystallization behaviour of thin films of various compositions of $\mathrm{GaGeTe}, \mathrm{Sb}_{2} \mathrm{Te}_{3}$ and $\mathrm{BiSe}$ These studies have shown that these materials can be good candidates for use as recording media in erasable phase-change optical recording.
\end{abstract}

Keywords. Optical recording; chalcogenide thin films; laser annealing.

\section{Introduction}

Optical recording is presently considered as a potential replacement for magnetic recording. This is not only because of the higher amount of information stored per unit area on the media surface in the optical recording, but also due to the absence of head crashes, wear and dust related problems encountered in the magnetic recording (Marchant 1990). Out of three optical recording, viz (i) ROM - read only memory, (ii) WORM - write once read many times and (iii) erasable optical recording, it is only the last one which can be a fully functional replacement for any existing magnetic recording systems. The two leading contenders of erasable optical recording at present are magneto-optic and phase change. Magneto-optic (MO) recording relies on a sharply thresholding thermal effect (Nagato et al 1986). Here, a laser beam heats the surface of an amorphous magnetic film above its Curie temperature. Subsequent cooling in the presence of an external magnetic field alters the magnetization direction of the heated spot in a predictable way. Data are erased by reversing the direction of the magnetic field applied. Reading is done using the Kerr or Faraday effect (Bloomberg and Connell 1988). The first magneto-optical recording layer was $\mathrm{Mn}-\mathrm{Bi}$ (Chen et al 1978). Later on attention was focussed on amorphous rare-earth transition metal alloys such as $\mathrm{Tb}-\mathrm{Fe}$, $\mathrm{Gd}-\mathrm{Co}, \mathrm{Gd}-\mathrm{Fe}, \mathrm{Ga}-\mathrm{Tb}-\mathrm{Fe}, \mathrm{Tb}-\mathrm{Dy}-\mathrm{Fe}$ and $\mathrm{Gd}-\mathrm{Fe}-\mathrm{Bi}$. While magneto-optic recording is presently the most matured technology it has few disadvantages in replacing the existing media. These include an optical head with lot of componentswhich makes the alignment complex--and a poor signal to noise ratio.

The most promising alternative to $\mathrm{MO}$ recording which has received considerable attention in recent years (Chiba et al 1993) is the erasable phase-change optical recording. Chalcogenide thin films are used as the recording medium in phase-change optical recording. The films are switched between amorphous and crystalline states using the heat of a focussed laser beam. Large reflectivity differences between amorphous and crystalline states are then used to store and retrieve information (Gravesteijn et al 
1989). An active chalcogenide layer should meet the following requirements: (i) Moderate thermal conductivity: If the film is highly conducting, the beam spreads to the neighbouring area leading to a low recording density. But a very low conductivity could make it difficult to quench the material for amorphization. Hence a moderate thermal conductivity is required.(ii) Low melting point: A low melting point material is preferred because it increases recording sensitivity. But if it is too low, it may give rise to hole formation during recording. (iii) High activation energy: As amorphous materials are metastable, high activation energy $\left(E_{\mathrm{a}}\right)$ ensures amorphous (data) stability. (iv) Large change in optical constants: There should be a large change in optical constants $(n, k)$ between the amorphous and crystalline states as this gives good optical contrast and improves signal to noise ratio. (v) Compatible alloy or compound formation: Actual atomic motion is involved in the repeated melting/quenching/annealing cycles in phase-change optical recording. Eventually a noticeable phase segregation will occur if the original alloy is not perfectly compatible. (vi) Density compatibility: If the two material forms have a large difference in density, then the recording process will induce strain within the film which could damage the film or force material to flow away from the data tracks. (vii) High absorption: The material. should have a high absorption coefficient $\left(>10^{4} \mathrm{~cm}^{-1}\right)$, so that incident laser energy can be utilized effectively.

Thus a material for use in phase change optical recording has to meet so many different simultaneous requirements. This restricts the choice of the materials that can be used. A survey of literature reveals that most of the work has been carried out on chalcogenide thin films having Te as a major constituent (Ovshinsky 1992). Some experiments on Se based materials (Barton et al 1986) and non-chalcogenide materials (Afonso et al 1992) have also been reported. An ideal recording medium has not yet been found and there is an active material research going on in this direction. The issues of long term data stability, fast crystallization/erasing, and the capability of being switched many times $\left(>10^{6}\right)$ between amorphous and crystalline states still continue to challenge physicists and material scientists. Studies were therefore undertaken with a view to try new materials as recording media. Keeping the basic requirements of recording medium in mind, we have investigated thin films of GaGeTe, SbTe and BiSe (Sripathi et al 1991, 1992; Reddy et al 1992).

\section{Experimental}

Bulk GaGeTe, SbTe and BiSe alloys were prepared by the melt quenching technique. Appropriate quantities of high purity (99.99\%) elements, sealed in a quartz ampoule under high vacuum conditions, were kept in a vertical furnace at appropriate temperatures $\left(500^{\circ} \mathrm{C}-1000^{\circ} \mathrm{C}\right)$ for about $36 \mathrm{~h}$ for homogeneous mixing, and were then quenched in cold water to avoid any segregation. Fine powder obtained by crushing of the bulk alloy was used for closed space sublimation. Thin films were deposited using a high vacuum $\left(\sim 10^{[-6]}\right.$ Torr $)$ coating unit on variety of substrates-glass, quartz, polished $\mathrm{Si}$ wafers, freshly cleaved $\mathrm{NaCl}$ and polymethylmethacrylate (PMMA). The composition of the bulk and the thin films was determined using a PHI 590 A scanning Auger microprobe and energy dispersive X-ray analysis. The film thickness was measured using a surface profiler Talystep (Talyor Hobson, UK). Transmittance and reflectance measurements were carried out using a Hitachi-330 double beam ultraviolet-visible-near 


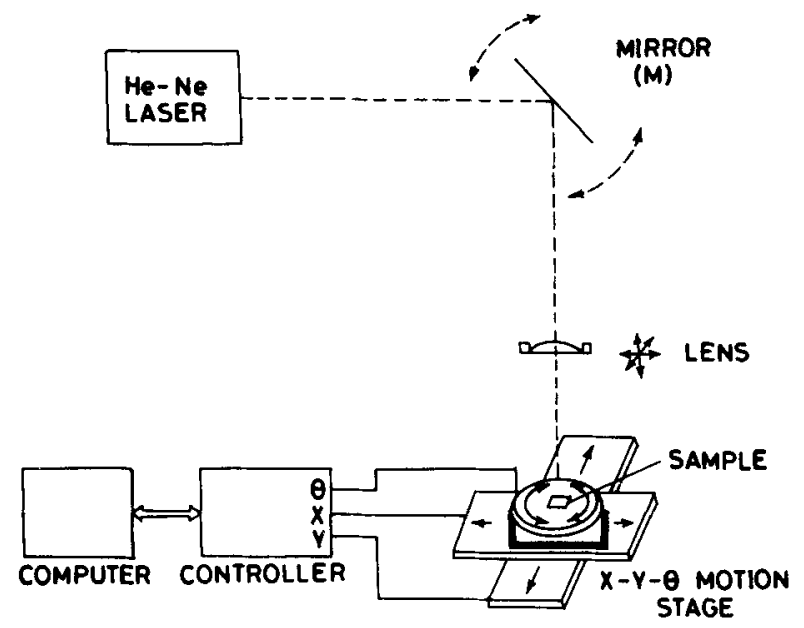

Figure 1. Schematic diagram of the laser annealing set-up.

infrared spectrophotometer. Surface morphology was studied using a scanning electron microscope (SEM; Philips 525 M). Transmission electron microscope (Philips $\mathrm{CM}-12$ ) has been used for selected area diffraction studies and to investigate the microstructure of the chalcogenide thin films. Glancing angle X-ray diffractometer was also used to analyze the structure of the as-deposited and annealed films. A Dupont 330 differential scanning calorimeter was used for determining $T_{\mathrm{g}}, T_{\mathrm{c}}$ and $T_{\mathrm{m}}$.

A computer controlled laser annealing arrangement was designed to anneal the films (figure 1). A CW He-Ne laser beam $(\lambda=632.8 \mathrm{~nm})$ with $25 \mathrm{~mW}$ peak output was used as the light source. The beam intensity was varied using optical density filters. The sample was mounted on an $\mathrm{X}-\mathrm{Y}-\Theta$ motion stage [Aerotech, ATS $100-100 \mathrm{~N}$ ] with a resolution of $0.1 \mathrm{~m}$ and 0.05 . These $\mathrm{X}-\mathrm{Y}-\Theta$ movements were controlled by a microprocessor unit (UNIDEX 12S-AEROTECH) having $64 \mathrm{~KB}$ memory and 32 key input device. Patterns of different specifications were generated using a personal computer and transmitted to control unit through RS-232C communication channel. The beam was aligned towards the lens $(f=7 \mathrm{~mm})$ with the help of a mirror ' $M$ ' and focussed by adjusting the vertical axis of the lens mounting assembly. The sample mounting assembly was enclosed in a pressurized glass chamber in order to avoid both contamination and oxidation. It was ensured with the help of a tilt stage that the sample surface is in the same horizontal plane (to an accuracy of $0.25 \mu \mathrm{m}$ ).

\section{Results and discussions}

Thin films of GaGeTe, SbTe and BiSe obtained by vacuum evaporation were smooth, coherent and pinhole free. Films of uniform thickness were obtained by optimizing the deposition parameters. Before carrying out any analysis, compositional analysis of the deposited films was done using scanning auger microprobe (SAM)/electron spectroscopy for compositional analysis (ESCA) and energy dispersive X-ray analysis (EDAX). The chemical composition of the films as determined by SAM/ESCA and EDAX were found to be nearly (within 2 at. \%) the same as that of the starting material. 


\subsection{Crystallization behaviour}

There are two approaches which have been used to study crystallization kinetics by differential scanning calorimetry: isothermal analysis (Ramanan and Fisher 1982), and the non-isothermal (Yinnon and Uhlmann 1983) analysis. Since many phase transformations occur too rapidly to be measured under isothermal conditions because of the transients inherently associated with the experimental apparatus, non-isothermal analysis has been preferred for the study of crystallization kinetics in the present work.

The approach to non-isothermal analysis is based on the description of isothermal transformations, because of the well developed mathematical analysis of the latter in literature. Isothermal crystallization processes in glasses are interpreted by the well known Johnson-Mehl-Avrami [JMA] equation (Christian 1975)

$$
x=1-\exp \left(-K t^{n}\right)
$$

where $x$ is the transformed volume fraction or the extent of crystallization, $t$ the time, $n$ the Avrami exponent [or order parameter/reaction coefficient (Tanabe et al 1989) indicating which mechanism dominates the phase change behaviour, crystal nucleation and/or its growth] and $K$ the rate constant which usually follows an Arrhenius relationship with temperature

$$
K=A \exp \left(E_{\mathrm{a}} / R T\right),
$$

where $A$ is pre-exponential factor and $E_{\mathrm{a}}$ activation energy. Hence the crystallization rate for isothermal annealing rate is

$$
\frac{\mathrm{d} x}{\mathrm{~d} t}=K n t^{n-1} \exp \left(-K t^{n}\right)
$$

In non-isothermal studies, the material is crystallized by heating from an amorphous state under the influence of a linear temperature $\left(T=T_{0}+\beta t, T_{0}=\right.$ initial temperature, $\beta=$ heating rate). Under such conditions, the crystallization process may be treated as a sequence of infinitesimally small isothermal crystallization steps (Dutta 1990). Hence the isothermal rate equation (3) should also be applicable for non-isothermal condition and takes the following form

$$
\frac{\mathrm{d} x}{\mathrm{~d} t}=n K t^{n-1}(1-x)
$$

Integration of (4) leads to

$$
\ln (1-x)=n K \int_{t_{0}}^{t} t^{n-1} \mathrm{~d} t
$$

By using (1), (2) and the relation $T=T_{0}+\beta t$, the above equation becomes

$$
\ln (1-x)=\frac{n A}{\beta^{n}} \int_{T_{0}}^{T} \exp \left(E_{\mathrm{a}} / R t\right)\left(T-T_{0}\right)^{n-1} \mathrm{~d} T .
$$


By taking logarithm on both sides

where

$$
\begin{aligned}
\ln [-\ln (1-x)] & =\ln \left[n A / \beta^{n} \int_{T_{0}}^{T} \exp \left(E_{\mathrm{a}} / R t\right)\left(T-T_{0}\right)^{n-1} \mathrm{~d} T\right], \\
& =\ln X(T)-n \ln \beta
\end{aligned}
$$

$$
X(T)=n A \int_{T_{0}}^{T} \exp \left(E_{\mathrm{a}} / R t\right)\left(T-T_{0}\right)^{n-1} \mathrm{~d} T .
$$

Thus the equation $\ln [-\ln (1-x)]=\ln X(T)-n \ln (\beta)$ can be used to find Avirami exponent $n$ by plotting $\ln [-\ln (1-x)]$ as a function of $\ln \beta$ at constant temperature (i.e. same temperature on different exotherms of a sample at different heating rates). Ozawa (1971) and others (Sestak 1974; Chen 1978) have applied JMA equation to non-isothermal analysis without any pre-condition/assumption. This was strongly disagreed by Henderson (1979), who mentioned that JMA equation can be applied only to those transformations, in which the nucleation process takes place early in the transformation and the nucleation rate is zero thereafter (this concept was referred to as site saturation (Cahn 1956)). Yinnon and Uhlmann (1983) summarized seven different treatments used in non-isothermal analysis, all based on (1) and (2) and the relation $T=T_{0}+\beta t$.

In the present work, JMA equation has been applied to non-isothermal analysis based on the following two assumptions: (i) nucleation both at surface and interior of the specimen is same, and (ii) the nucleation rate per unit volume in the entire specimen depends only on instantaneous temperature.

Figure 2 shows the typical thermogram of $\mathrm{Ga}_{5} \mathrm{Ge}_{19} \mathrm{Te}_{76}$ in an inert gas atmosphere using differential scanning calorimeter (DSC). The thermogram was taken using the

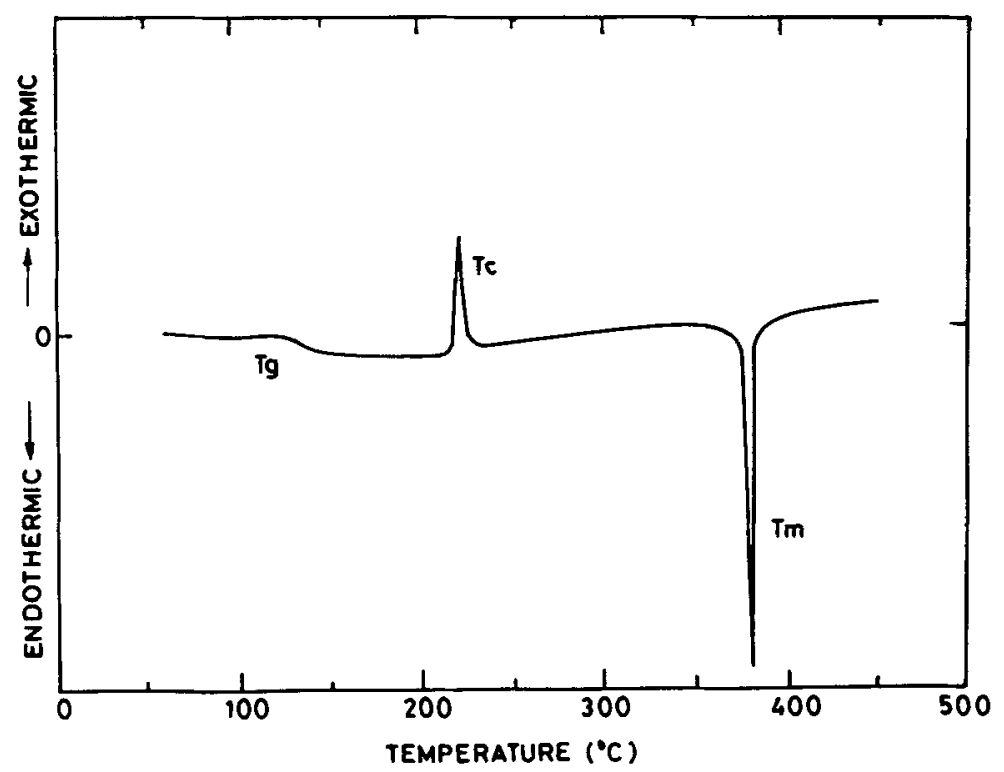

Figure 2. DSC thermogram indicating the temperatures of glass transition $\left(T_{\mathrm{B}}\right)$, crystallization $\left(T_{\mathrm{c}}\right)$ and melting $\left(T_{\mathrm{m}}\right)$. 
Table 1. Values of activation energy $\left(E_{\mathrm{a}}\right)$ and avrami exponent $(n)$ for GaGeTe, SbTe and BiSe films of different composition.

\begin{tabular}{lll} 
& $E_{\mathrm{a}}(\mathrm{eV})$ & $n$ \\
\hline $\mathrm{Ga}_{5} \mathrm{Ge}_{19} \mathrm{Te}_{76}$ & 0.9 & 1.20 \\
$\mathrm{Ga}^{20} \mathrm{Ge}_{30} \mathrm{Te}_{50}$ & 2.58 & 1.66 \\
$\mathrm{Bi}^{20} \mathrm{Se}_{90}$ & 1.20 & $1 \cdot 37$ \\
$\mathrm{Bi}{ }^{20} \mathrm{Se}_{80}$ & 1.51 & 1.37 \\
$\mathrm{Bi}{ }_{50} \mathrm{Se}_{50}$ & 1.05 & 1.45 \\
$\mathrm{Sb}_{2} \mathrm{Te}_{3}$ & 2.00 & 1.80 \\
\hline
\end{tabular}

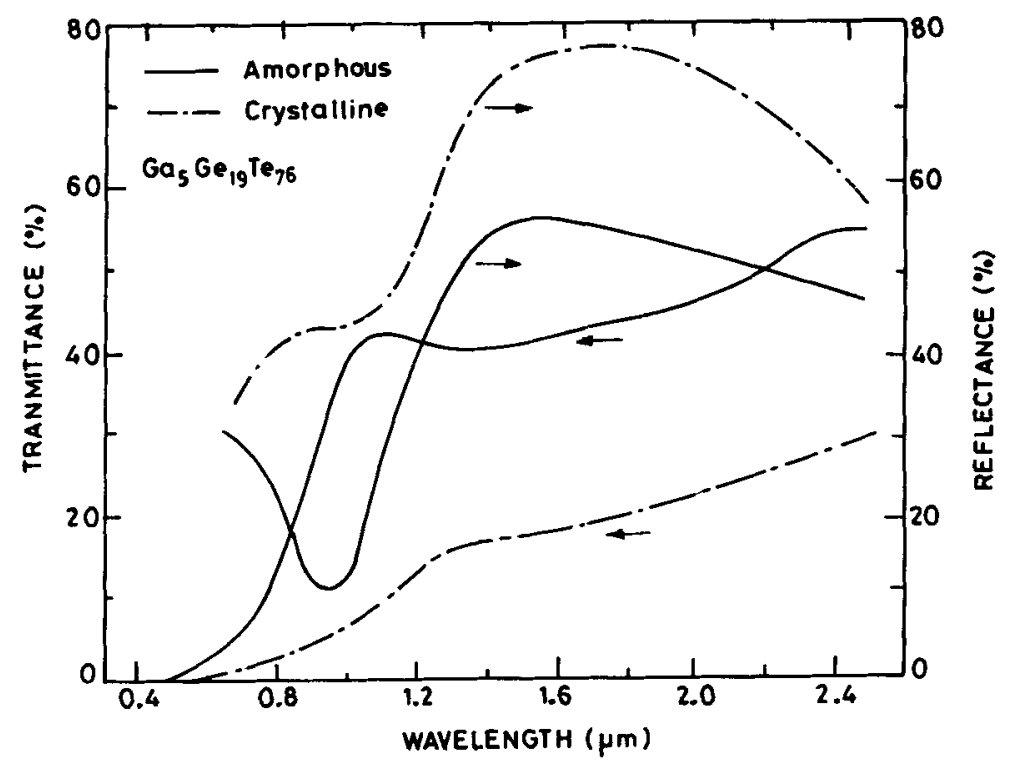

Figure 3. Optical transmittance and refiectance spectra of as-deposited (amorphous) and annealed (crystallized) films of $\mathrm{Ga}_{5} \mathrm{Ge}_{19} \mathrm{Te}_{76}$ as a function of wavelength.

film material scrapped off the substrate. The DSC scans showed a small shift in the baseline at $127^{\circ} \mathrm{C}$ which is indicative of the glass transition. The exothermic peak observed for $\mathrm{Ga}_{5} \mathrm{Ge}_{19} \mathrm{Te}_{76}$ (in the same thermogram) at $222^{\circ} \mathrm{C}$ is a signature of the crystallization temperature. The sample after exhibiting the exothermic peak, was heated again and no exothermic peak was observed up to $222^{\circ} \mathrm{C}$ which ensured complete crystallization in the first cycle. It is assumed that the interface temperature is negligibly affected by the liberation of the latent heat. Calorimetric measurements were made under non-isothermal conditions and a multiscan technique has been used for crystallization studies. The activation energy was calculated using the Kissinger's (1957) formula. It has been shown, that the dependence of $T_{\mathrm{p}}$ (peak temperature) on $\beta$ is given by

$$
-\ln \left(\beta / T_{\mathrm{p}}^{2}\right)=E_{\mathrm{a}} / R T_{\mathrm{p}}-\text { const. }
$$

The activation energy $E_{\mathrm{a}}$ of crystallization can thus be evaluated from the slope of the plot of $\ln \beta / T_{\mathrm{p}}^{2}$ vs $10^{3} / T_{\mathrm{p}}$. The $E_{\mathrm{a}}$ and $n$ values for various films are given in table 1 . 


\subsection{Optical properties}

For optical recording media where a high absorption of the incident radiation is desired, we are mainly concerned with $\alpha>10^{3} \mathrm{~cm}^{-1}$ and hence in band to band transitions only. Figure 3 shows the measured optical transmittance and reflectance of an as-deposited amorphous film of $\mathrm{Ga}_{5} \mathrm{Ge}_{19} \mathrm{Te}_{76}$ films of thickness $1000 \AA$. Transmittance ( $T$ ) starts increasing at around $0.8 \mu \mathrm{m}$ followed by a rapid rise, correspondingly there is a sharp fall in reflectance $(R)$. The minimum in reflectance at $0.95 \mu \mathrm{m}$ is due to interference as confirmed by a shift in minima with film thickness (figure 4). Change in film thickness from 1150 to $1500 \AA$ results in the shift of minimum from $1.08 \mu \mathrm{m}$ to $1.38 \mu \mathrm{m}$. The optical constants $n$ and $k$ were determined using transmittance, reflectance and thickness data with the help of a computer program based on the NewtonRaphson method; the calculated $n$ and $k$ for GaGeTe films are shown in figure 5 . The $n$ values are comparatively higher than $k$ values reflecting the semiconducting behaviour. The $k$ values increase gradually with decreasing wavelength. The high $k$ values reveal high absorption coefficient $\alpha=(4 \pi k / \lambda)$, variation of $\alpha$ with energy is shown in figure 6. The optical gap determined by extrapolating the linear portion of $(\alpha h v)^{1 / 2}$ vs $h v$ curve to X-axis (where $(\alpha h v)^{1 / 2}$ is equal to zero) was $0.85 \mathrm{eV}$, which is in the range of $E_{\mathrm{g}}$ 's of semiconductors used for optical recording. Figure 3 also shows the measured $R$ and $T$ spectra of $\mathrm{Ga}_{5} \mathrm{Ge}_{19} \mathrm{Te}_{76}$ films after crystallizing by thermal annealing. The strong interference reflectance minimum appearing at $0.95 \mu \mathrm{m}$ in as-deposited $\mathrm{Ga}_{5} \mathrm{Ge}_{19} \mathrm{Te}_{76}$ films disappeared after annealing. These changes in transmittance and reflectance values are attributed to the change in the optical constants only, as we did not observe

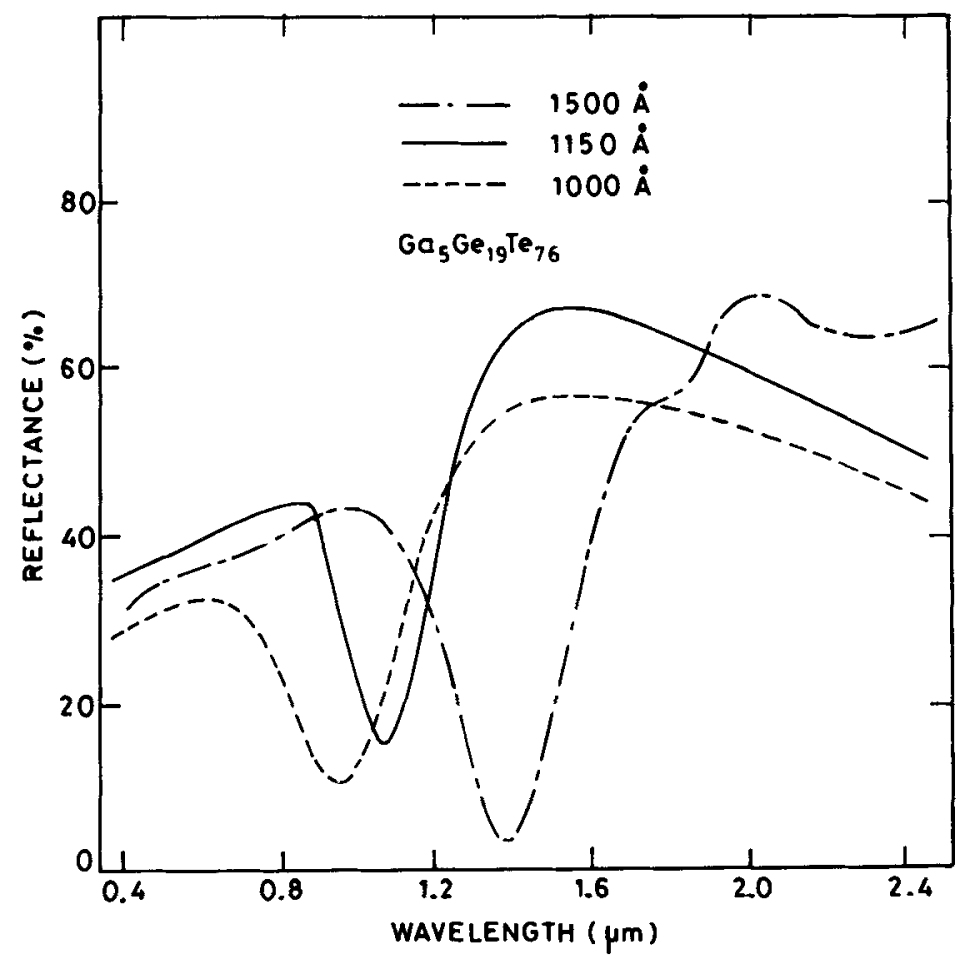

Figure 4. Shift of reflectance minima with film thickness of $\mathrm{Ga}_{5} \mathrm{Ge}_{19} \mathrm{Te}_{76}$ films. 


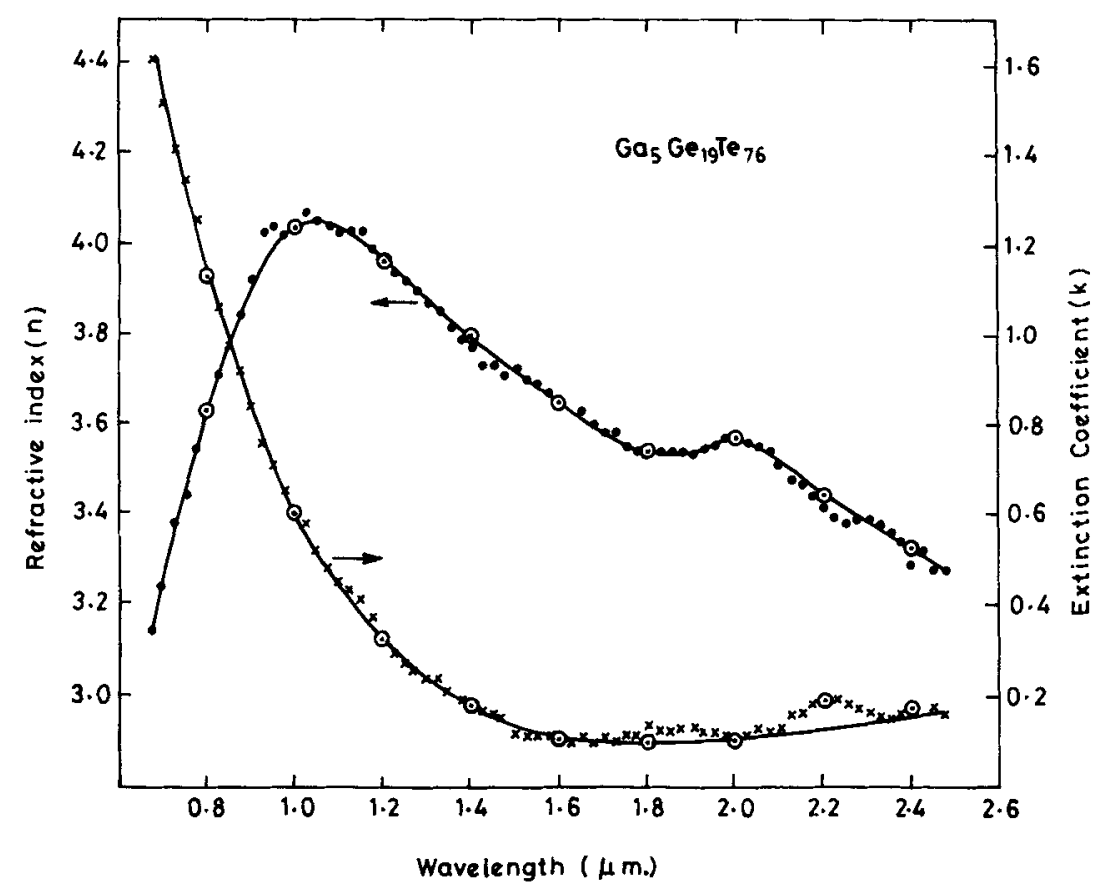

Figure 5. Optical constants of as-deposited $\mathrm{Ga}_{5} \mathrm{Ge}_{19} \mathrm{Te}_{76}$ films.

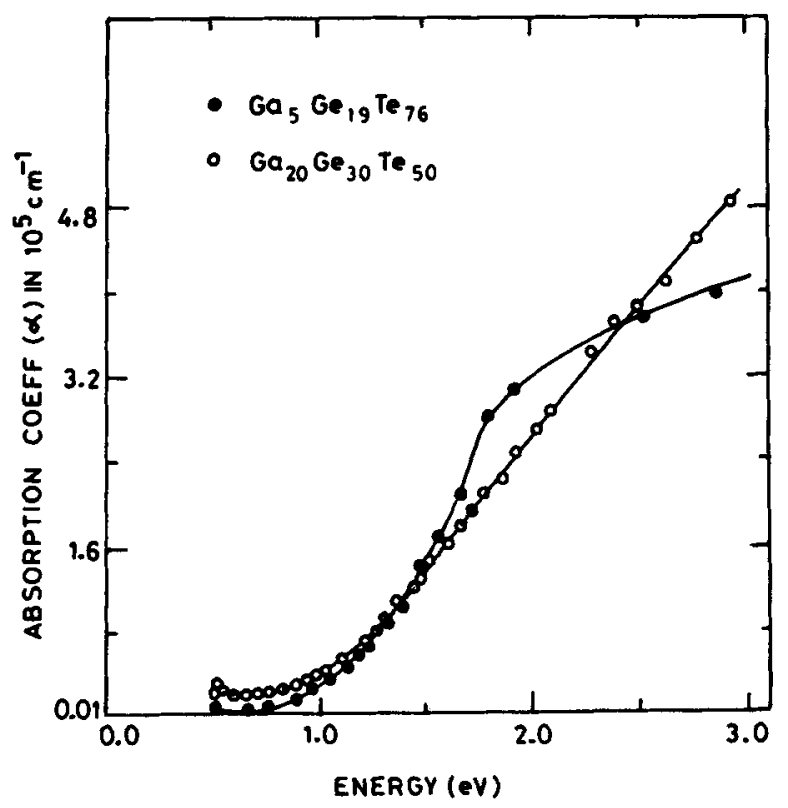

Figure 6. Spectral dependence of optical absorption coefficient of as-deposited GaGeTe films. 
any change in film thickness after crystallization. In order to determine the change in reflectance on annealing, the optical contrast ratio was determined. The optical contrast ratio is defined as (Rhee et al 1987)

$$
\text { contrast ratio }=\left(R_{\mathrm{c}}-R_{\mathrm{a}}\right) / R_{\mathrm{c}},
$$

where $R_{\mathrm{c}}$ is the reflectance of the crystalline phase and $R_{\mathrm{a}}$ the reflectance of the amorphous phase. Measured optical contrast at $0.83 \mu \mathrm{m}$ was 0.46 . The film thickness has been optimized, in order to get the maximum contribution due to interference effects (Vander Poel et al 1988) at a desired wavelength. The optimum thickness for $\mathrm{Ga}_{5} \mathrm{Ge}_{19} \mathrm{Te}_{76}$ films was $0.1 \mu \mathrm{m}$. Similar studies have been made on $\mathrm{Bi}_{10} \mathrm{Se}_{90}, \mathrm{Bi}_{20} \mathrm{Se}_{80}$, $\mathrm{Bi}_{50} \mathrm{Se}_{50}$ and $\mathrm{Sb}_{2} \mathrm{Te}_{3}$ films. Our experimental results show that (i) $\mathrm{GaGeTe}, \mathrm{Sb}_{2} \mathrm{Te}_{3}$ and BiSe films exhibit high absorption coefficient $\left(>10^{4} \mathrm{~cm}^{-1}\right)$ in the visible region, (ii) an optical contrast ratio of about 0.2 at $830 \mathrm{~nm}$ (the wavelength of the diode laser normally employed in optical recording), can be obtained in $\mathrm{GaGeTe}, \mathrm{Sb}_{2} \mathrm{Te}_{3}$ and $\mathrm{BiSe}$ films, (iii) amorphous phases of these films are stable and the optical properties of asdeposited films remain unaffected by normal environmental conditions. This was clear from the optical spectra of films taken after seven months (the films were kept under normal environmental conditions during this period).

The above results suggest that $\mathrm{GaGeTe}, \mathrm{Sb}_{2} \mathrm{Te}_{3}$ and BiSe films are good contenders for phase-change optical recording media.

The major limitation to the method of reflection measurement is imposed by the state of the surface of the materials. It is thus of great importance to ensure that surface layers of the material are characteristic of the bulk of the material, and are not contaminated by the oxide layer. Some tellurium rich films have been reported (Carcia et al 1988) to get oxidized under normal environmental conditions. But the stability, in optical properties of the GaGeTe films, observed by us suggest that they are not prone to oxidation.

For both amorphous and crystalline $\mathrm{GaGeTe}, \mathrm{Sb}_{2} \mathrm{Te}_{3}$ and BiSe films, in the range of wavelengths investigated, $n$ is several times higher than unity while $k$ is about unity or less. Thus $n$ and $k$ values of these films represent the semiconducting behaviour. Our result indicate that the band gap of $\mathrm{Bi}_{x} \mathrm{Se}_{1-x}$ films decreases with increasing $\mathrm{Bi}$ concentration. The $a$-Se band gap reported by several workers (Chopra 1973) is $2.02 \mathrm{eV}$, and the $E_{\mathrm{g}}$ values obtained by us are $1.6 \mathrm{eV}, 0.95 \mathrm{eV}$ and $0.25 \mathrm{eV}$ for $\mathrm{Bi}_{10} \mathrm{Se}_{90}$, $\mathrm{Bi}_{20} \mathrm{Se}_{80}$ and $\mathrm{Bi}_{50} \mathrm{Se}_{50}$ respectively. The bond strengths of $\mathrm{Bi}-\mathrm{Bi}, \mathrm{Bi}-\mathrm{Se}$ and $\mathrm{Se}-\mathrm{Se}$ bonds are $47.9,67$ and $79 \mathrm{kcal} / \mathrm{mol}$ respectively. It is therefore expected that with the addition of ' $\mathrm{Bi}$ ' to 'Se' the optical band gap should drop continuously (particularly when miscibility between $\mathrm{Bi}$ and $\mathrm{Se}$ is high). It may be mentioned that $E_{\mathrm{g}}$ for $\mathrm{Bi}_{2} \mathrm{Se}_{3}$ as reported (Bhattacharya et al 1982) is $0.32 \mathrm{eV}$, in agreement with our observations.

\subsection{Laser annealing}

Laser annealing of amorphous films and the resultant transition to crystalline state (Ovshinsky 1970) has been investigated extensively because of its potential importance to optical data storage. Some important questions that need to be addressed before laser annealing is carried out are:

(i) What is the wavelength of the laser required?

(ii) What is the optimum beam intensity for a particular effect to occur in a controlled manner? 
(iii) How long should the beam dwell on the irradiated site?

(iv) What is the resulting temperature distribution? and

(v) How to avoid or minimize the unwanted effects in the sample?

In order to answer the above questions, a complete knowledge of the laser source chosen for annealing the sample as well as the sample's optical and thermal properties is required.

The choice of the laser wavelength is dictated by the spectral absorption coefficient of the recording film. The most important characteristics of the laser source (Noguchi 1982) are that the beam should be circular and must permit a diffraction limited spot. For this, the laser must operate with a fundamental mode beam $\mathrm{TEM}_{00}$, which results in smallest beam divergence. The angle of divergence is given by

$$
\phi(\text { in radians })=2 \lambda / \pi d,
$$

where $\lambda$ is the wavelength and $d$ the diameter of the limiting aperture through which the beam has passed. Typical $\phi$ values for the $\mathrm{He}-\mathrm{Ne}$ laser are in the range $0 \cdot 2-1$ and as high as 10 for the high powered pulsed lasers, e.g. ruby or $\mathrm{Nd}$ : glass lasers. The laser beam can be focussed to a spot approaching the wavelength of the light itself. In fact, the wavelength defines the fundamental limit imposed by diffraction theory and one can achieve a spot of this size by using the highest quality of focussing optics. Though diode lasers are attractive for optical recording systems, they have longer wavelengths and higher divergence as compared to argon ion or $\mathrm{He}-\mathrm{Ne}$ lasers. Due to shorter wavelength and small spot size, significantly greater data density is possible with argon ion lasers. However argon ion laser is comparatively expensive and has a shorter life with attendant cooling problems. Taking the above factors into consideration (and since our work was concerned only with feasibility studies), $\mathrm{He}-\mathrm{Ne}$ laser has been used in the present studies primarily because it has a relatively low wavelength, it can be operated in $\mathrm{TEM}_{00}$ mode, and $25 \mathrm{~mW}$ power is sufficient for obtaining required temperatures in chalcogenide glasses. The operation cost is very minimum as there is no need for cooling arrangement.

The theoretical calculations for temperature rise and its distribution in a material due to a laser beam irradiation are based on conventional heat diffusion equation for a stationary laser beam as follows:

$$
\frac{\partial T}{\partial t}-D \nabla^{2} T=\frac{Q}{C_{\mathbf{p}}},
$$

where $Q$ is the source power density, $D$ the thermal diffusivity and $C_{\mathrm{p}}$ the specific heat per unit volume.

We have used the approach proposed by Cline and Anthony (1977) in which the temperature $T$ at a point $x, y, z$ (figure 7 ) is given by

$$
T(x, y, z)=\frac{P}{C_{\mathrm{p}} D R} f(x, y, z, v),
$$

where the function $f$ is temperature distribution function formed due to the superposition of discrete Gaussian sources of a moving laser beam,

$$
f=\int_{0}^{\infty} \frac{\exp \{-H\}}{\left(2 \pi^{3}\right)^{1 / 2}\left(1+\mu^{2}\right)} \mathrm{d} \mu .
$$




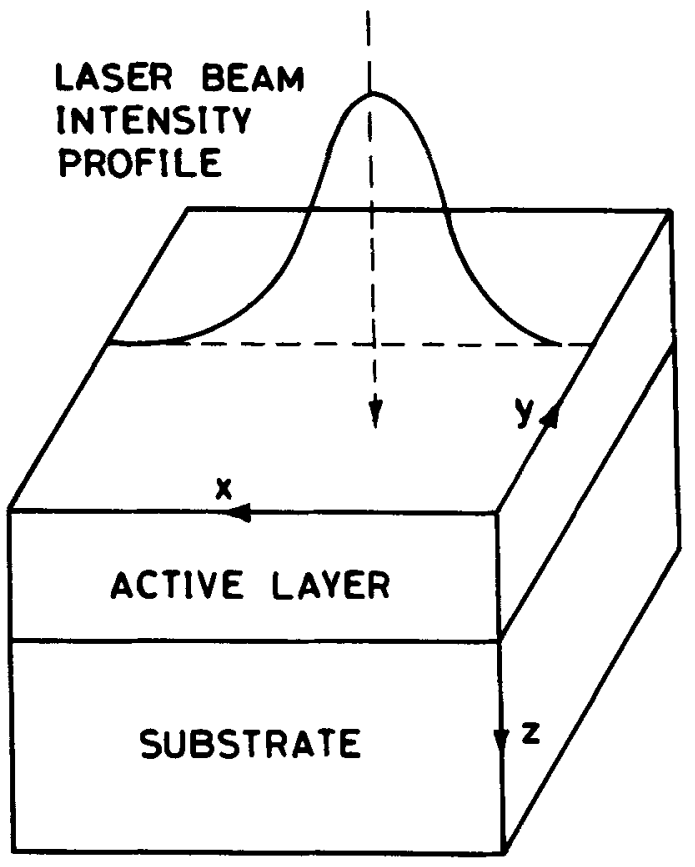

Figure 7. Schematic diagram illustrating laser-beam heating of a thin film moving in the $\mathrm{X}$-direction.

where

$$
\begin{aligned}
& H=\frac{\left(X+\frac{1}{2} p \mu^{2}\right)^{2}+Y^{2}}{2\left(1+\mu^{2}\right)}+\frac{Z^{2}}{2 \mu^{2}}, \\
& \mu^{2}=\frac{2 D t^{\prime}}{R}, \rho=\frac{R V}{D}, X=\frac{x}{R}, Y=\frac{y}{R}, Z=\frac{z}{R} .
\end{aligned}
$$

The temperature distribution profile along the $\mathrm{x}$-axis was calculated from (9). Optical reflection losses of the film were taken inio account for estimating the power absorbed. The effects of convective and radiative heat losses are too small for the time periods involved here and hence are neglected. The calculated profile as a function of normalized distance from the centre of the laser beam is shown in figure 8 for different scan speeds of $\mathrm{Ga}_{5} \mathrm{Ge}_{19} \mathrm{Te}_{76}$ films. As is evident, the temperature profile as a function of normalized position in the active layer is roughly Gaussian in shape for lower velocity of $v=0.1 \mathrm{~cm} / \mathrm{sec}$. But as the scanning speed is increased, the temperature profile deviates significantly from the Gaussian shape and assumes an asymmetric shape with respect to $X=0$. Maximum temperature at the surface $(Z=0)$ of the film (with $D=0.0005 \mathrm{~cm}^{2} / \mathrm{sec}$ and $C_{\mathrm{p}}=1.4 \mathrm{~W} / \mathrm{cm}^{2}{ }^{\circ} \mathrm{C}$ ) is $467^{\circ} \mathrm{C}$. With increasing scan speed the peak temperature decreased; this is because of the lower dwell time of the laser beam on the sample. Temperature distribution profiles at surface were also calculated for $\mathrm{Ga}_{20} \mathrm{Ge}_{30} \mathrm{Te}_{50}, \mathrm{Sb}_{2} \mathrm{Te}_{3}$ and $\mathrm{Bi}_{x} \mathrm{Se}_{1-x}(x=0 \cdot 1,0 \cdot 2$ and $0 \cdot 5)$ thin films as a function of normalized distance from centre of the laser beam for different scan speeds. The maximum temperature attained is governed by both the thermal constants and optical 


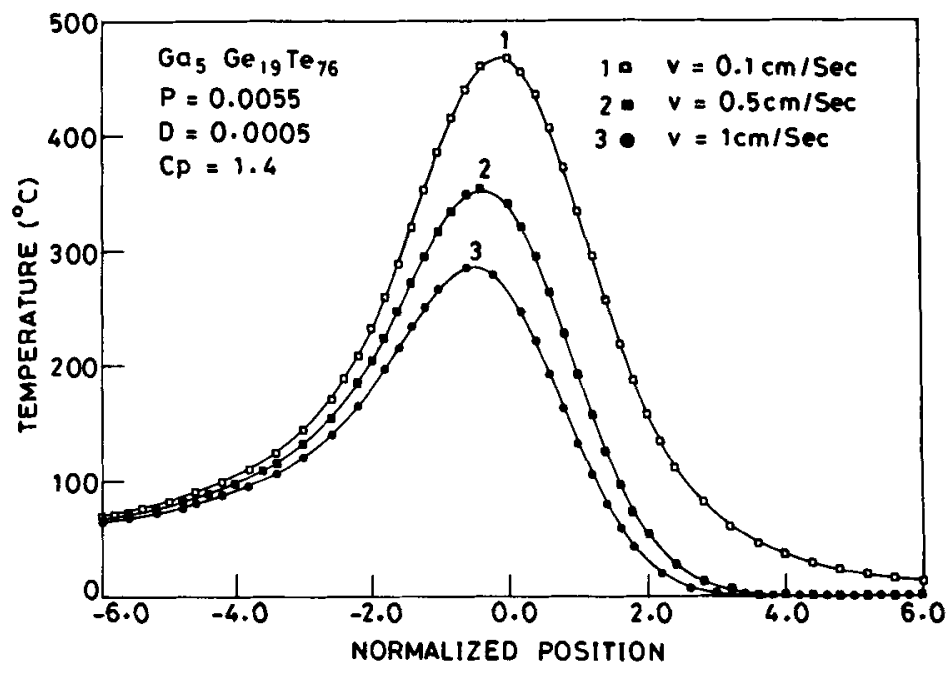

Figure 8. Calculated temperature profiles vs the normalized distance for $\mathrm{Ga}_{5} \mathrm{Ge}_{19} \mathrm{Te}_{76}$ films for different scanning velocities.

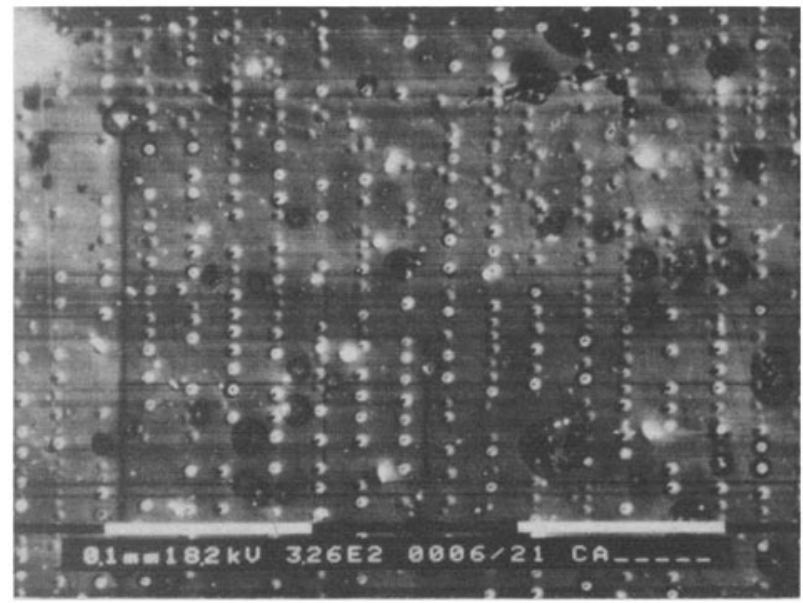

Figure 9. SEM topograph of a typical laser annealed GaGeTe film indicating partial ablation.

properties, but at a given velocity, temperature distribution profiles are similar in all the compositions studied.

Since the thickness of the films used in the present study varied from 400 to $800 \mathrm{~A}$, the temperature change was insignificant at different depths of the sample.

The sample for laser annealing was mounted on a translational stage as described in experimental set-up (figure 1). Laser light was focussed so that high power densities are obtainable with a relatively low power $\mathrm{He}-\mathrm{Ne}$ laser. The as-deposited amorphous films of area $1.5 \times 1.5 \mathrm{~cm}^{2}$ were scanned under the focussed laser beam. The singlet lens used in our experiment can produce a spot of $5.6 \mu \mathrm{m}$ with proper alignment. However, because of either stage wobbling, or sample unevenness the beam may not be focussed 
Table 2. Energy densities at different dwell periods used in the experiment.

\begin{tabular}{lccc}
\hline Region & $\begin{array}{c}\text { Scan speed } \\
(\mathrm{mm} / \mathrm{sec})\end{array}$ & $\begin{array}{c}\text { Dwell time } \\
(\mathrm{m} \cdot \mathrm{sec})\end{array}$ & $\begin{array}{c}\text { Energy density } \\
\left(\mathrm{J} / \mathrm{cm}^{2}\right)\end{array}$ \\
\hline i & 2 & $2 \cdot 150$ & 5.375 \\
ii & 3 & 1.430 & 3.575 \\
iii & 4 & 1.075 & 2.687 \\
iv & 5 & 0.680 & 2.150 \\
v & 6 & 0.716 & 1.790 \\
vi & 7 & 0.614 & 1.535 \\
vii & 8 & 0.537 & 1.342 \\
viii & 9 & 0.477 & 1.192 \\
ix & 10 & 0.430 & 1.075 \\
\hline
\end{tabular}

Intensity $=I=P / r_{0}^{2}$, where $P$ is the power of the beam on the lens (as measured by flux meter) $=18 \mathrm{~mW}$

that accurately. Owing to the above mentioned experimental difficulties, the spot radius in the present study has been maintained to $15 \mu \mathrm{m}$. The separation between the two successive scanned lines is varied from $4 \mu \mathrm{m}$ to $10 \mu \mathrm{m}$ depending on the purpose of the study. For a particular beam intensity, depending upon the scan speed, the effects of the laser beam on the sample could be conveniently classified into four regions: (a) unaffected, (b) crystallized, (c) melted and (d) ablated. For higher scanning speeds ( $>10 \mathrm{~mm} / \mathrm{sec}$ ), there was not much time for the beam to cause topographical or structural changes. And for very low scanning speeds $(2-4 \mathrm{~mm} / \mathrm{sec})$ the films were ablated, a typical SEM picture is shown in figure 9. Thus a threshold in laser exposure can be reached which separates solid-state annealing from melting of the active layer. Table 2 shows the energy densities at different dwell periods used in the experiment.

Based on the theoretically obtained temperature profiles, the scan speed was adjusted so that the film is annealed above $T_{\mathrm{g}}$ and below $T_{\mathrm{m}}$ for crystallization to occur. Crystallization of the films was confirmed by TEM (figure 10).

For optical studies, the separation between the neighbouring lines was maintained at $5 \mu \mathrm{m}$ and the films were scanned with a spot size of $15 \mu \mathrm{m}$ so that the entire film gets annealed because of the overlap of the successive scanned lines. Reflectivity changes were compared for different scanning speeds and it was observed that: in GaGeTe films, reflectivity changes occur with scan parameters corresponding to iii-v regions (as mentioned in table 2). Similarly for $\mathrm{Bi}_{10} \mathrm{Se}_{90}$ and $\mathrm{Bi}_{20} \mathrm{Se}_{80}$ films, $15 \%$ reflectivity change was observed with parameters mentioned in the regions iv-vi. For $\mathrm{Bi}_{50} \mathrm{Se}_{50}$, reflectivity contrast was not observed.

Temperature modelling gave a good agreement with experimental results in the region of ablation and crystallization (low to moderate scan speeds). But at high scan speed $(1 \mathrm{~cm} / \mathrm{sec})$ there is slight discrepancy between the theoretically expected and the experimentally observed effects. For example for $\mathrm{Ga}_{5} \mathrm{Ge}_{19} \mathrm{Te}_{76}$ films the maximum temperature obtained at $v=1 \mathrm{~cm} / \mathrm{sec}$ was $280^{\circ} \mathrm{C}$-a temperature at which the film should get crystallized (DSC and structural studies), but no crystallization was observed at this scanning speed by laser beam heating. However, as evident from the temperature distribution profile the crystallization temperature interval is within a narrow range and outside this range both theoretical values and experimental observed effects are in agreement. The slight discrepancy at $v=1 \mathrm{~cm} / \mathrm{sec}$ is attributed to 

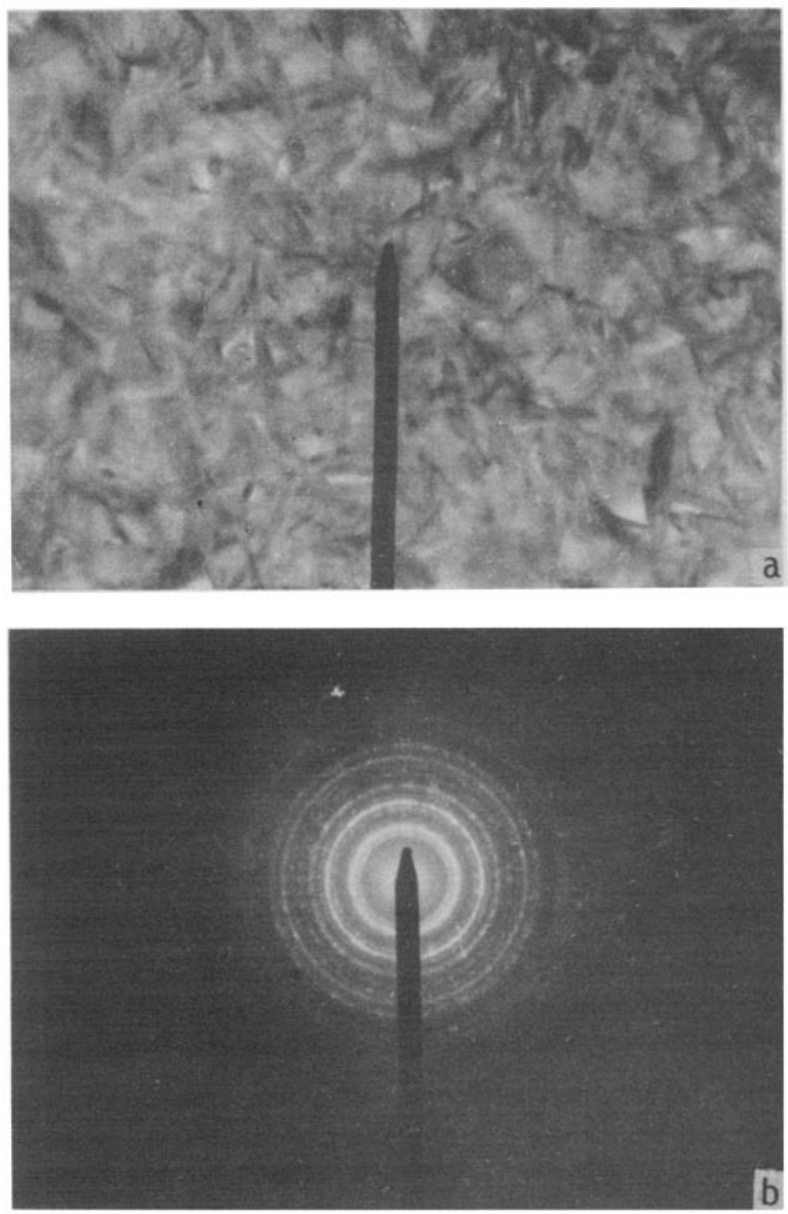

Figure 10. a. Bright field transmission electron micrograph of a laser annealed GaGeTe film and $\mathbf{b}$. the selected area diffraction.

either error in the material parameter chosen or experimental difficulties in observing these minute effects, or both. A comparison of the data presented in table 2 with the energy densities reported in literature (Rubin et al 1987) suggests that the energy densities available can be used for both writing and erasing of a phase change optical recording disk.

\section{References}

Afonso C N, Salis J and Calatind F 1992 Appl. Phys. Lett. 603123

Barton R, Charles R D, Rubin K and Grace L 1986 Appl. Phys. Lett. 481255

Bhattacharya R N and Pramanik P 1982 J. Electro. Chem. 129332

Bloomberg D and Connell G 1988 Magneto-optical recording (eds) C Mee and E Daniel (New York: McGraw Hill) Vol. 3

Cahn J W 1956 Acta Metall. 4 449, 573

Carcia P F, Kalk F D and Swartz D G 1988 J. Appl. Phys. 641671

Chen H S 1978 J. Non-Cryst. Solids 27257 
Chiba R, Yamazaki H, Yagi S and Fujimori S 1993 Jap. J. Appl. Phys. 32834

Chopra K L (ed.) 1973 Optical behaviour of amorphous materials, in Optical behaviour of materials (Delhi: Thomson Press Ltd.)

Christian 1975 Theory of phase transformation of metals and alloys (New York: Pergamon Press)

Cline H E and Anthony R. T 1977 J. Appl. Phys. 483895

Dutt A 1990 Polym. Commun. 31 451

Gravesteijn D J, Vander Poel, Scholte P M L O and Van Uijen C M J 1989 Philips Tech. Rev. 44250

Henderson D W $1979 \mathrm{~J}$. Thermal Anal. 15325

Marchant A 1990 Optical recording-A technical review (Reading, Massachusetts: Addison-Wesley)

Nagto K, Kawamoto A, Sato T and Yorozu T 1986 J. Appl. Phys. 633856

Noguchi M 1982 Appl. Opt. 212665

Ovshinsky S R 1970 US Patent \# 3, 530, 441

Ovshinsky S R 1992 J. Non-Cryst. Solids 141203

Ozawa T 1971 Polymer 12150

Ramanan V R V and Fisher G E 1982 J. Appl. Phys. 532273

Reddy G B, Dhar A, Malhotra L K and Sharmila E K 1992 Thin Solid Films 220111

Sestak J 1974 Phys. Chem. Glasses 6137

Sripathi Y, Reddy G B and Malhotra L K 1991 J. Mater. Sci.: Materials in Electronics 2109

Sripathi Y, Reddy G B and Malhotra L K 1992 J. Mater. Sci.: Materials in Electronics 3164

Tanabe T, Tetsuo 1 and Funakoshi N 1989 Jap. J. Appl. Phys. 28 L1845

Vander Poel C J, Gravesteijn D J and Van Uijen C M J 1988 J. Appl. Phys. 591819

Yinnon H and Uhlmann D R 1983 J. Non-Cryst. Solids 54253 